\title{
EDITORIAL
}

\section{PATRIMONIO PÚBLICO Y LIBRE ACCESO A LA CIENCIA}

\author{
Joaquín Rodríguez López \\ Director de Contenidos Digitales. \\ Residencia de Estudiantes. Consejo Superior de Investigaciones Científicas. Madrid.
}

El problema es en principio sencillo de contar y de comprender, y seguramente consiga concitar un acuerdo mayoritario con un ejemplo actual: aun cuando gravite sobre nosotros el riesgo de una posible pandemia de gripe aviaria, no existe, que se sepa, una base de datos en la que se hayan recogido los casos de contagio en humanos registrados en China o Vietnam, y menos frecuentes y fidedignas son aún las referencias sobre las aves de corral y sus avatares actuales. Contamos con los testimonios que reúne la World Organisation for Animal Healht ${ }^{1}$, pero los especialistas ya han señalado que sus datos son incompletos, que son geográficamente deficientes, que contienen errores y que, además, no poseen información genética de ningún tipo o, cuando la poseen, cuando la secuenciación aparece, es fragmentaria o parcial, guardada a buen recaudo por algún gobierno que la usufructa y monopoliza en su único provecho o administrada y explotada por un pequeño grupo de investigadores vinculados a organizaciones como la $U S$ Centres for Disease Control and Prevention $^{2}$, la World Health Organisation ${ }^{3}$ o la $\mathrm{FAO}^{4}$. No cabe duda alguna que el bien común, en este como en tantos otros casos, debe anteponerse al legítimo afán comercial, y que no cabe excepción o salvedad cuando de vidas humanas se trata, que no existe justificación alguna para no poner al servicio de la comunidad científica los conocimientos necesarios para que la suma de los esfuerzos genere una inteligencia superior a la de los científicos aislados y se logre, así, una vacuna o un antiviral que prevengan o combatan la enfermedad. Así lo entienden, por ejemplo, aquellos científicos que ponen su conocimiento y sus descubrimientos a disposición de quienes puedan o quieran utilizarlos, publicándolos en la página de GenBank ${ }^{5}$, por ejemplo, en vez de incluir y difundir sus datos a través de redes privadas o de pago, redes restringidas, por tanto, inaccesibles para la mayor parte de la comunidad científica, sobre todo para aquélla que dispone de menores recursos financieros y sufre, sin embargo, las mayores amenazas de plagas, pandemias y enfermedades ${ }^{6}$. El Instituto Zooprofilattico Sperimentale delle Venezie ${ }^{7}$ ha publicado en GenBank, anteponiendo el bien común a cualquier consideración académica o económica, todos los datos que obran en su poder relativos al genoma del virus $\mathrm{H} 5 \mathrm{~N} 1^{8}$, lo que no sólo no mermará el prestigio académico de quien lo haya hecho ni disminuirá un ápice el reconocimiento de su autoría intelectual, sino que potenciará ambos y, de paso, rendirá un servicio impagable a la humanidad.

Internet, las redes de comunicación y los soportes digitales han convulsionado la manera de producir, publicar, editar, difundir, comunicar y reproducir los contenidos 
de cualquier índole, en este caso que nos ocupa los de naturaleza científica, haciendo factible que cualquiera pueda potencialmente transmitir algún contenido, que puedan tejerse redes de conocimientos compartidos y que pueda prescindirse de los intermediarios tradicionales mediante la reapropiación y uso de las nuevas herramientas de comunicación. Es difícil implantar una cadena causal que establezca si fue antes la necesidad o la herramienta, el precisar de canales de comunicación propios e independientes para el intercambio fluido de conocimientos o la Internet y toda la panoplia de programas y plataformas que permiten a cualquier usuario medio avezado construir y administrar su propio sitio. La tipología y variedad es tan amplia, que me restringiré a mencionar alguno de los proyectos de open source para la publicación de contenidos en la web: Open Journal System ${ }^{9}$ o Hyperjournal ${ }^{10}$ pueden ser utilizados para la edición de revistas de contenidos científicos con facilidad; en el mundo del open access todo se desarrolla de manera que la intercomunicación y la colaboración sean factibles y que el conocimiento derivado se incremente, de ahí que se hayan desarrollado dos estándares, uno que pretende incrementar la interoperabilidad entre los archivos para facilitar una diseminación amplia y eficiente de los contenidos $\left(\mathrm{OAI}^{11}\right)$, y otro que persigue enlazar todas las referencias bibliográficas contenidas en cualquier artículo, de manera que el encadenamiento mutuo genere un verdadero árbol de conocimientos y experiencias compartidas $\left(\right.$ OPCIT $\left.^{12}\right)$; abunda, también, la oferta de software libre para la generación y administración de sitios web, desde SPIP $^{13}$ hasta PHPNuke $^{14}$; Bioline Internacional ${ }^{15}$ (como la Budapest Open Access Initiative o como Highwire Press ${ }^{16}$ ) proporciona, además, apoyo a las instituciones y revistas de países en vías de desarrollo para facilitar y hacer asequible la publicación y difusión de sus conocimientos $^{17}$.

Esa independencia radical y repentina viene acompañada, además, de un nuevo conjunto de licencias que permiten al productor de los contenidos facultar el uso, difusión, reproducción y potencial modificación del contenido por él producido, sin renunciar por ello, en ningún caso, al reconocimiento de autoría o graduando el alcance de los permisos concedidos. La nueva ordenación jurídica de la propiedad intelectual que propone Creative Commons ${ }^{18}-\mathrm{y}$ su derivado para el caso que nos ocupa, Science Commons $^{19}$ - no es exactamente una forma radical de copyleft por negación total del copyright sino, más bien, el tipo de ordenamiento que conviene a un mundo fluido $\mathrm{y}$ circulante, donde las ideas y los contenidos son segregados, compartidos y manipulados por millones de personas en cuyas manos queda siempre la potestad de hacer más o menos accesible y modificable el contenido de su creación. El copyright ${ }^{20}$ pertenece a un mundo estático, el del papel, y las estrecheces y restricciones que le son connaturales no son de este nuevo mundo ${ }^{21}$, aunque quepa, cómo no, seguir utilizándolo y aplicándolo cuando el creador así lo desee, pero no cuando el creador lo rechace. La digitalización ubicua de los contenidos y el nuevo sistema dúctil y flexible de derechos de autor no hacen otra cosa, por tanto, que potenciar y fortalecer los imperativos categóricos inscritos en el campo científico. Todo aquel que desee seguir la carrera científica deberá observar tres exigencias que, aunque no se enuncien ni se juramenten explícitamente, son de obligado cumplimiento si no se desea recibir la sanción y censura de la comunidad científica: la lógica del descubrimiento y el debate por el establecimiento de la verdad, en primer lugar, deben regirse por métodos contrastables basados en la discusión racional y en la exclusión de la violencia o el ejercicio directo del poder y, en segundo lugar, no deben plegarse en ningún caso a intereses ajenos a los del propio campo científico, es decir, a intereses comerciales o partidistas que puedan desviarlos de sus objetivos intrínsecos. Dos hechos recientes apuntalan esta afirmación: el primero es masivo, el segundo aparentemente individual: en una 
encuesta publicada en el año 2005 por la revista Nature $^{22}$, se puso al descubierto que el $33 \%$ de los encuestados habían cometido o incurrido durante los últimos tres años alguno de los diez comportamientos reprobables que eran motivo de la pesquisa (cambiar el diseño, la metodología o los resultados de un estudio en respuesta a las presiones de las entidades financiadoras o patrocinadoras y pasar por alto el uso o la cuestionable interpretación de datos defectuosos o incompletos, sobre todo), y ese proceder reprochable aumentaba hasta el $38 \%$ cuando se trataba de científicos que estaban haciendo su carrera, que se veían impelidos de alguna manera a manipular el resultado de sus trabajos en supuesto beneficio de su integración plena en la comunidad. Los imperativos categóricos existen porque se manifiestan en forma de autocensura y autoreconvención, casi sin necesidad de que el campo científico aplique sus correctivos; el caso aparentemente individual es, ya lo presentirán, una manifestación de autocondena de gran calado mediático fruto de la presencia intangible pero definitiva de ciertos imperativos éticos, la del doctor Hwang Woo Suk y el fraude de la clonación celular ${ }^{23}$. Cabría añadir una exigencia más a los dos principios enunciados, una exigencia que tiene mucho que ver con el interés general y la difusión pública y gratuita del conocimiento científico: es imposible silenciar, ocultar o encubrir los resultados de una investigación, no darlos a conocer o hacerlo sólo de manera restringida y partidista, sin que la comunidad científica sancione este comportamiento como antinatural, como impropio y opuesto a los principios fundamentales de funcionamiento del campo científico, porque la ciencia se fundamenta sobre un sedimento de altruismo y filantropía que apela siempre íntimamente a la conciencia del científico. La difusión, comunicación y circulación del conocimiento, por tanto, dentro de la misma comunidad científica pero, también fuera de ella, son preceptos seguramente inefables pero no por ello menos perentorios y apremiantes. No se trata, simplemente, de que medrar en la carrera científica exija la publicación continua de materiales de muy heterogénea calidad sino de, más fundamentalmente, que los resultados de las discusiones por el establecimiento de una verdad determinada dentro del campo científico exigen ser publicados, hechos públicos, para que cobren realidad y legitimidad. Hacer ciencia es sinónimo, por tanto, de hacer públicos sus resultados, o investigar, dicho de otra manera, es publicar y si esa demanda incontrovertible se ve respaldada por un incremento exponencial de la visibilidad y de los índices de impacto ${ }^{24}$ cuando se publica en una revista de acceso abierto y lo que podría estar abocado a quedar sepultado en la avalancha de artículos y novedades editoriales queda ahora indizado, ordenado y clasificado -tal como hace DOAJ ${ }^{25}$ o, también, Google Scholar ${ }^{26}-\mathrm{y}$, por tanto, reconocible y accesible, no hay nada más sencillo que hacer lo que hay que hacer.

No se trata de ser maniqueo y sostener que todo aquel que difunde pública y gratuitamente sus contenidos se sujete completamente y sin resquicios a la lógica altruista del campo científico, pero lo que sí parece cierto es que cuando interfieren motivaciones económicas, comerciales o profesionales, competitivas, en el trabajo científico, caben muchas más posibilidades de que los procedimientos y los resultados de las investigaciones hayan sido manipulados o pervertidos en beneficio de una lógica ajena al campo. Por eso no hay que tomar a broma o percibir como mero escarceo el movimiento internacional, capitaneado por los mismos científicos $^{27}$, que promueve simultáneamente la publicación y difusión abiertas del conocimiento científico generado con fondos públicos -devolver a la comunidad en forma de conocimiento depurado lo que la comunidad concedió en forma de recursos brutos- y el libre e irrestricto acceso a la ciencia y sus resultados -engrandeciendo y fortaleciendo a la propia comunidad científica y, en consecuencia, a los saberes que de ella se deriven-. 
La relación cronológica de declaraciones, movimientos y proyectos institucionales más relevantes por el libre acceso a la ciencia, para aquellos que quieran formarse una opinión más fundamentada, pasa por la Budapest Open Access Initiative ${ }^{28}$ promovida por George Soros, nada sospechoso de impulsar algaradas anarquistas; la Bethesda Statement on Open Access Publishing ${ }^{29}$, una de las primeras manifestaciones públicas a este respecto no azarosa ni casualmente emitida por el Howard Hughes Medical Institute en Chevy Chase, Maryland, Estados Unidos, algo más que una escuela de curanderos; la Declaración de Berlín ${ }^{30}$ promulgada en la primera Open Access Conference y amparada por el Max-Planck-Institute , $^{31}$, una institución que no se dedica, precisamente, a los ecos de sociedad; los principios recogidos en el Free Access to Science S2 $^{32}$ amparados y divulgados por la cuarta universidad del mundo ${ }^{33}$, Standford, que no es, exactamente, una academia de costura; el punto primero de la Declaración de Principios ${ }^{34}$ de la Primera Cumbre Mundial de la Sociedad de la Información ${ }^{35}$, que no fue solamente una reunión de amiguetes, donde se establece y reclama lo siguiente:

Nosotros, los representantes de los pueblos del mundo, reunidos en Ginebra [...] con motivo de la primera fase de la Cumbre Mundial sobre la Sociedad de la Información, declaramos nuestro deseo y compromiso comunes de construir una Sociedad de la Información centrada en la persona, integradora y orientada al desarrollo, en que todos puedan crear, consultar, utilizar y compartir la información y el conocimiento, para que las personas, las comunidades y los pueblos puedan emplear plenamente sus posibilidades en la promoción de su desarrollo sostenible y en la mejora de su calidad de vida, sobre la base de los propósitos y principios de la Carta de las Naciones Unidas y respetando plenamente y defendiendo la Declaración Universal de Derechos Humanos.
Si he utilizado cierto tono irónico en la relación institucional anterior es porque es demasiado frecuente encontrarse con descalificaciones integrales al movimiento open access tildándolo de irrelevante, marginal, sedicioso e, incluso, extremista, que su calidad de contenidos es desdeñable e incontrastable. Me permitirán el desahogo.

Cabría hacer, también, una breve historia de la publicación o edición abierta en línea -y convendría ir diciendo, de paso, que en la web se desdibujan o se hacen imprecisos, por una parte, los límites entre los medios que en el mundo analógico se diferencian claramente y, por otra, el mismo acto de la edición de un contenido, que ahora se convierte en cualquier acción destinada a la publicación en línea- aunque, más que un criterio cronológico, convendría seguir el de relevancia: la que pasa por ser la primera publicación en línea abierta fue el repositorio arXiv.org ${ }^{36}$, que recogía prepublicaciones de campos científicos muy autónomos, los de la física y las matemáticas, para añadir después los de la informática y la biología cuantitativa. Publicar o editar dejaba de ser un conjunto de actos encaminados a la impresión exclusiva en papel y su sentido se amplía hasta alcanzar el de la divulgación y difusión de un contenido a través de la web mediante el autoarchivado o la autoedición. A ese experimento pionero y todavía en funcionamiento hay que añadir, sin duda, el de PLOS (la Public Library of Science) ${ }^{37}$, un conjunto de publicaciones de libre acceso, que se amparan en las licencias de Creative Commons, y que revolucionaron el mundo editorial al invertir varios procedimientos editoriales bien establecidos, el principal de los cuales fue el pago por publicación, es decir, es el grupo de investigación que publica los resultados de sus trabajos quienes subvencionan y financian su publicación y comunicación, corolario lógico si se tiene en cuenta que las investigaciones fueron desarrolladas con fondos públicos y revierten, de esa manera, a la comunidad que las costeó. A propósito: las cabeceras de PLOS son 
medicina, biología, biología computacional, genética y patógenos, un espejo en el que deberíamos mirarnos. Scielo, red en la que se encuentra la Revista española de Salud Pública $^{38}$, fue un proyecto pionero radicado en América Latina y que hoy ha saltado a este lado del mar con el propósito, en sus propias palabras, de agrupar a «un conjunto de publicaciones periódicas científicas, especializadas en ciencias de la salud, proporcionando el acceso a toda la colección de revistas, a los números individuales, así como al texto completo de cada uno de los artículos». Por fin una red de conocimientos compartidos abierta y gratuita que reparte su protagonismo y alcance con otra iniciativa española promovida por el CINDOC y el portal Tecnociencia, e-revist@ $\mathrm{s}^{39}$.

Que no le engañen, el problema, después de todo lo dicho, es fácil de comprender: la mayoría de nosotros recibe financiación pública para desarrollar sus trabajos de investigación, descubrimientos que deben regresar a la sociedad de la que derivan en forma de conocimiento compartido y de aplicaciones derivadas, y ese imperativo de comunicación y visibilidad puede cumplirse sobradamente utilizando los canales de difusión y publicación que nos proporciona Internet y las aplicaciones de software gratuitas que gestionan y administran ese contenido, todo ello sin que se pierda un ápice de protagonismo, relevancia o reconocimiento intelectual porque existen instrumentos jurídicos nuevos que nos permiten graduar el acceso a esos contenidos de la manera que dispongamos y porque no hay razón alguna para que lo publicado y diseminado en red no pueda sufrir un proceso de evaluación y acreditación académicas tan estricto y riguroso como el que soportan las publicaciones en papel, al contrario, la visibilidad de sus trabajos, su penetración y su impacto, tal como demuestran todos los estudios, se incrementarán notablemente, exponencialmente, al tiempo que presta un servicio insustituible a sus semejantes y contribuye a que el bien común sea el rico fundamento de nuestra convivencia.
No se lo piense más, no se amedrante, apoye el acceso libre a la ciencia, únase a la red libre de conocimiento global que ya se está promoviendo en la Universidad de Standford o en la Biblioteca Pública de la Ciencia o, también, en la Open Access Initiative y publique sus contenidos usted mismo o a través de los editores que sigan los principios del libre acceso al conocimiento.

\section{BIBLIOGRAFÍA}

1. World Organization for animal health. Disponible en: OIE http://www.oie.int/eng/en_index.htm

2. Centers for diseases control and prevention. Department of health and human services. Disponible en: http://www.cdc.gov/

3. World Health Organization. Disponible en: http://who.org

4. Organización de la Naciones Unidas para la agricultura y alimentación. Disponible en: http:// www.fao.org/index_es.htm

5. Gen Bank Overview. National Center for biothechnology information. Disponible en: http:// www.ncbi.nih.gov/Genbank/index.html

6. Several countries blocking international bird flu research http://news.yahoo.com/s/afp/ 20060303/hl_afp/healthflunetherlands_ 060303172832;_ylt=A86.I04zTBZE1G4BTRITO7gF;_ylu=X3oDMTA5bGVna3NhBHN1YwNz c3JlbA--

7. Istituto Zooprofilattico Sperimentale delle Venezie. Disponible en:http://www.izsvenezie.it/dnn/

8 http://www.ncbi.nlm.nih.gov/entrez/viewer. fcgi? $\mathrm{db}=$ nucleotide $\&$ val $=88604738$

9. Public Knowledge Project. The University British Columbia. Disponible en: http://pkp.sfu.ca/ojs/

10. Hyperjournal web site. Disponible en: http:// www.hjournal.org/.

11. Open Archives Initiative. Disponible en: http:// www.openarchives.org/index.html

12. OpCit. The open citation project. Disponible en: http://opcit.eprints.org/ 
13. Spip. Sistema de Publicación por internet. Disponible en: http://www.spip.net/es

14. PhpNuke. Disponible en: http://phpnuke.org/

15. Bioline International. Disponible en: http:// www.bioline.org.br/

16. Highwire Press. Stanford University. Disponible en: http://highwire.stanford.edu/publishers/support.dtl

17. Rodríguez J. Ciencia y comunicación científica: edición digital y libre acceso al conocimiento, en

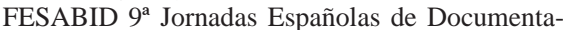
ción. Madrid: Infogestión; 2005. Disponible en: http://www.fesabid.org/madrid2005/descargas/pre sentaciones/actividades/rodriguez_joaquin.pps

18. Creative Commons España. Disponible en: http:// es.creativecommons.org/

19. Science Commons. Disponible en: http://science. creativecommons.org/

20. http://www.copyright.com/

21. En Free Culture http://free-culture.org/freecontent/

22. Brian C. Martinson, Melissa S. Anderson y Raymond de Vries. Scientist behaving badly. Nature 2005; 435:737-8. Disponible en: http://www.nature.com/nature/journal/v435/n7043/full/435737a.ht $\mathrm{ml}$

23. Microsiervos. Disponible en: http://www.microsiervos.com/archivo/ciencia/fraude-investigacionclonacion-corea-del-sur.html

24. Harnad S, Brody T. Comparing the Impact of Open Access (OA) vs. Non-OA Articles in the Same Journals. En: D-Lib Magazine, Volumen 10, Número 6, junio. http://www.dlib.org/dlib/june04/ harnad/06harnad.html

25. Directory of open acces journals. Universidad de Lund. Disponible en http://www.doaj.org
26. Google scholar beta. Disponible en: http://scholar.google.com/schhp?hl=en\&lr=

27. Millán JA. La revuelta de los científicos. Disponible en: http://jamillan.com/revuelta.htm

28. Budapest Open Acces Initiative. Budapest; 2002. Disponible en:http://www.soros.org/openaccess

29. Bethesda Statement on Open Access Publishing. Released June 20, 2003. Disponible en: http:// www.earlham.edu/ peters/fos/bethesda.htm

30. Berlin Declaration on Open Access to Knowledge in the Sciences and Humanities. Berlín; 2003. Disponible en: http://www.zim.mpg.de/openaccessberlin/berlindeclaration.html

31. Max Planck Gesellschaft. Disponible en: http://www.mpg.de/

32. Free acces to science. Disponible en: http:// www.dcprinciples.org/

33. Webometrics ranking of World Universities. Disponible en: http://www.webometrics.info/top 3000.asp.htm

34. International Telecommunication Union. http:// www.itu.int/dms_pub/itu-s/md/03/wsis/doc/S03WSIS-DOC-0004!!MSW-S.doc

35. World summit on the information society. Disponible en: http://www.itu.int/wsis/documents/doc_ multi-en-1161|1160.asp

36. Cornell University Library. Ar Xiv. Org Disponible en: http://arxiv.org/

37. Plublic Library of Science. Disponible en: http:// www.plos.org/

38. Scielo España. Instituto de Salud Carlos III. http://scielo.isciii.es/scielo.php

39. e-revistas. Portal Tecnociencia. Ministerio de Educación y Ciencia. Disponible en: http://www.tecnociencia.es/e-revistas/ 